\title{
TRANSFERÊNCIA DO MÚSCULO TRÍCEPS PARA BÍCEPS EM PACIENTES COM LESÃO CRÔNICA DO TRONCO SUPERIOR DO PLEXO BRAQUIAL
}

\author{
MUSCLE TRANSFER FROM TRICEPS TO BICEPS IN PATIENTS WITH CHRONIC \\ INUURY OF THE UPPER TRUNK OF THE BRACHIAL PLEXUS
}

Fabiano Inácio de Souza', Mateus Saito ${ }^{2}$, Luiz Koiti Kimura ${ }^{2}$, Rames Mattar Júnior ${ }^{3}$, Arnaldo Valdir Zumiotti ${ }^{4}$

\section{RESUMO}

Objetivo: Avaliar os resultados da transposição do tríceps para a flexão do cotovelo em pacientes portadores de lesão crônica e completa do tronco superior do plexo braquial. Métodos: Estudo retrospectivo, com inclusão apenas de pacientes que apresentassem bíceps grau 0 e tríceps grau 5 , submetidos à transferência anterior do músculo tríceps, operados entre 1998 e 2005. Foram pesquisados o lado acometido, o sexo, o tipo de acidente, a força de flexão do cotovelo, as complicações e a satisfação do pacientes, em 11 casos. Resultados: 10 pacientes eram do sexo masculino; a idade variou de 24 a 49 anos, com média de 33,7 anos. O tempo mínimo entre a lesão e o procedimento cirúrgico foi de 21 meses (variando de 21 a 74 meses). O lado esquerdo foi acometido em oito casos, enquanto o direito apenas em três. Obtiveram-se bons resultados em 10 pacientes, que adquiriram força de flexão do cotovelo grau 3 (dois casos) e grau 4 (oito casos), enquanto um evoluiu desfavoravelmente, com força grau 2. Dois casos evoluíram com complicações (síndrome compartimental inicial e tensionamento insuficiente). Todos os pacientes definiram-se como satisfeitos com o procedimento. Conclusão: A transposição anterior do músculo tríceps proporcionou satisfação dos pacientes em todos os casos, exceto um, obtendo-se forças grau 4 em oito casos, grau 3 em dois casos e grau 2 em um caso.

Descritores - Sistema musculoesquelético; Plexo braquial; Cotovelo

\section{ABSTRACT}

Objective: To evaluate the transposition of the triceps for elbow flexion in patients with chronic and complete injury to the upper trunk of the brachial plexus. Methods: Retrospective study, including only patients who had biceps grade 0 and triceps grade 5, who underwent anterior transfer of the triceps muscle, operated between 1998 and 2005. The affected side, sex, type of accident, strength of elbow flexion, complications and satisfaction of patients, were studied in 11 cases. Results: 10 patients were male, aged 24 to 49 years, with a mean of 33.7 years. The minimum time between injury and surgery was 21 months (range 21-74 months). The left side was affected in eight cases, the right only in three. Good results were obtained in 10 patients, who acquired grade 3 (two cases) and grade 4 (eight cases) strength in elbow flexion, while one evolved unfavorably with grade 2 strength. Two cases had complications (initial compartment syndrome and insufficient tensing). All patients reported satisfaction with the procedure. Conclusion: The anterior transposition of the triceps muscle provided patient satisfaction in all cases except one, attaining strength grade 4 in eight patients, grade 3 in two cases and grade 2 in one case.

Keywords - Musculoskeletal system; Brachial plexus; Elbow

\footnotetext{
1 - Doutorando e Mestre em Medicina pelo Instituto de Ortopedia e Traumatologia do HC-FMUSP.

2 - Médicos Assistentes do Grupo de Mão e Microcirurgia do Instituto de Ortopedia e Traumatologia do HC-FMUSP.

3 - Professor Associado da Faculdade de Medicina e Chefe do Grupo de Mão do Instituto de Ortopedia e Traumatologia do HC-FMUSP.

4 - Professor Titular do Departamento de Ortopedia e Traumatologia da Faculdade de Medicina da USP.
}

Trabalho realizado no Instituto de Ortopedia e Traumatologia do Hospital das Clínicas da Faculdade de Medicina da Universidade de São Paulo. Correspondência: Rua Dr. Ovídio Pires de Campos, 333, $3^{\circ}$ andar, Cerqueira César - 05403-010 - São Paulo, SP. E-mail: fabianoinacio@usp.br 


\section{INTRODUÇÃO}

As lesões traumáticas do plexo braquial acometem principalmente adultos jovens, em plena atividade produtiva, ocasionando déficit funcional variável e, muitas vezes, irreversível. Os acidentes motociclísticos são os principais responsáveis pelo aumento do número de pacientes. Segundo a Abraciclo ${ }^{(1)}$, as vendas de motocicletas no Brasil aumentaram de 460 mil unidades por ano, em 1998, para 1,8 milhões, em 2008.

O tratamento ideal das lesões do plexo braquial são realizados na fase subaguda, por meio de neurólises, interposições de enxertos e/ou neurotizações. Nestes casos é possível obter restauração da flexão do cotovelo em $60 \%$ dos $\operatorname{casos}^{(2)}$. Na fase crônica, os resultados com estas técnicas são desanimadores. Recomenda-se a utilização de transferências musculotendíneas, destacando-se flexoplastia de Steindler ${ }^{(3,4)}$, transferências do grande dorsal $^{(5)}$, peitoral maior e tríceps ${ }^{(6,7)}$, além dos transplantes musculares ${ }^{(8,9)}$.

O objetivo do presente estudo é avaliar os resultados da transposição do tríceps para a flexão do cotovelo em pacientes portadores de lesão crônica e completa do tronco superior do plexo braquial.

\section{MATERIAIS E MÉTODOS}

Foi realizado estudo retrospectivo, em que foram incluídos apenas pacientes que apresentassem bíceps grau 0 e tríceps grau 5 (Tabela 1), submetidos a transferência anterior do músculo tríceps, operados entre 1998 e 2005, por cirurgiões do Grupo de Mão e Microcirurgia do IOT-HCFMUSP. Foram pesquisados o lado acometido, o sexo, o tipo de acidente, a força de flexão do cotovelo, as complicações e a satisfação dos pacientes.

Este último quesito foi avaliado por meio da seguinte pergunta: "Você ficou satisfeito com esta cirurgia?”, em que a resposta poderia ser "sim” ou "não".

Tabela 1 - Graduação da força muscular

\begin{tabular}{c|c}
\hline Grau 0 & Ausência de contração muscular \\
\hline Grau 1 & Contração muscular, sem movimento articular \\
\hline Grau 2 & Movimento sem vencer a gravidade \\
\hline Grau 3 & Movimento que vence a gravidade \\
\hline Grau 4 & Movimento que vence a gravidade e alguma resistência \\
\hline Grau 5 & Força muscular normal \\
\hline Fonte: Seddon $\mathrm{HJ}^{(10)}$ &
\end{tabular}

\section{TÉCNICA CIRÚRGICA}

1 - Os pacientes eram dispostos em decúbito dorsal, sendo realizada incisão posterolateral no terço distal do braço;

2 - Dissecção por planos, identificação e proteção do nervo ulnar, individualização do tendão do tríceps com dissecção proximal;

3 - Secção da inserção do tríceps no olécrano com dissecção proximal da musculatura (Figuras 1 e 2);

4 - Incisão anterior no cotovelo, identificação do tendão do bíceps e dissecção do subcutâneo com direção posterolateral; e

5 - Transposição anterior do tendão do tríceps, suturando-o ao tendão do bíceps (Figura 3).

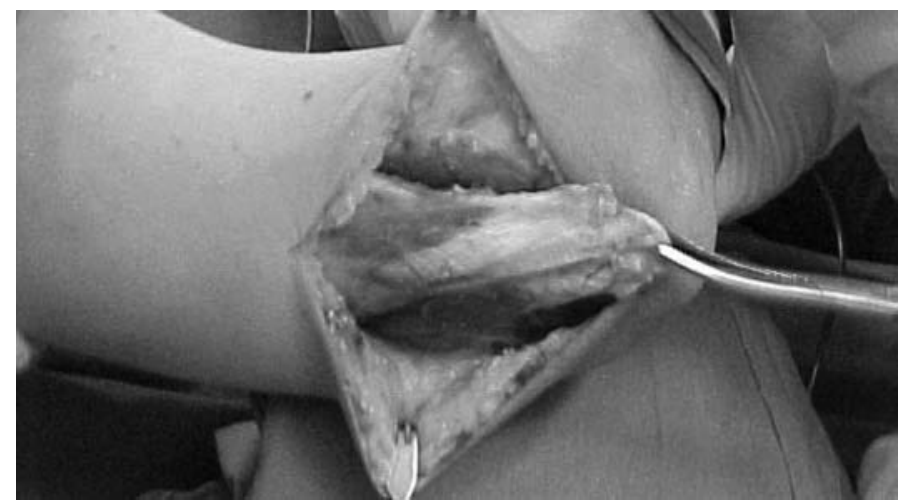

Figura 1 - Secção da inserção do tríceps no olécrano

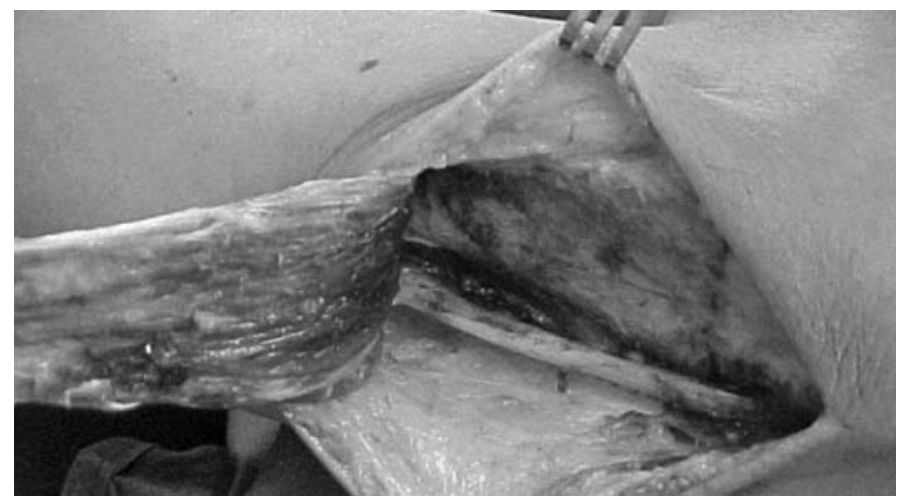

Figura 2 - Dissecção proximal do tríceps, individualizando-o do úmero e do nervo ulnar

\section{RESULTADOS}

Entre 1998 e 2005, foi operado um grupo de 11 pacientes, sendo 10 do sexo masculino; a idade variou de 24 a 49 anos, com média de 33,7 anos. O tempo mínimo entre a lesão e o procedimento cirúrgico foi de 21 meses (variando de 21 a 74 meses). Os acidentes motociclísticos causaram lesões em 10 pacientes e queda de altura 


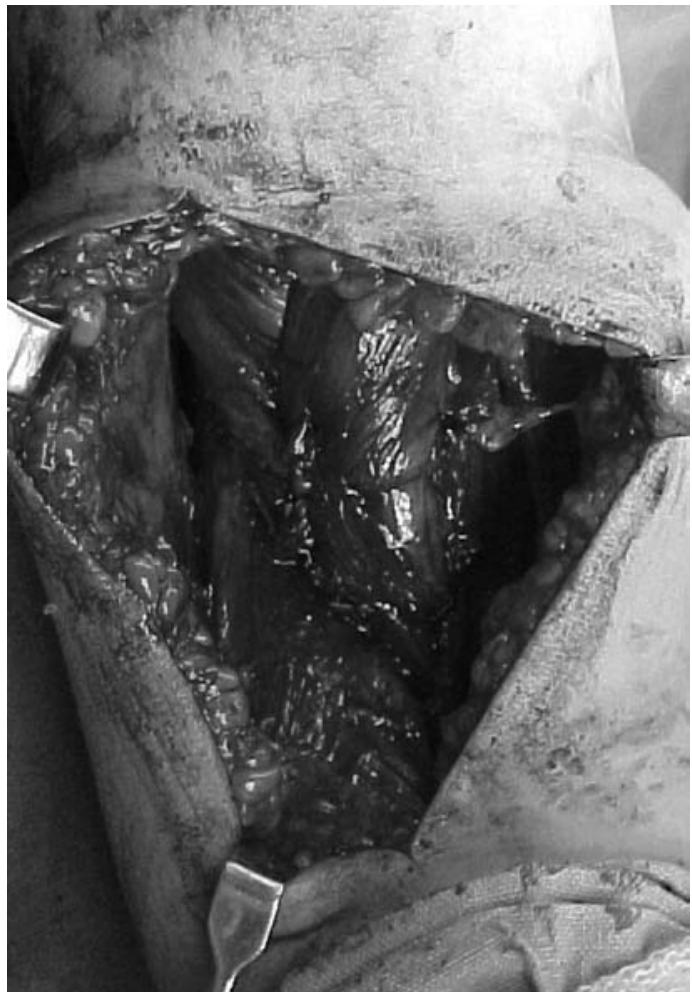

Figura 3 - Sutura do tríceps ao tendão do bíceps

em um caso. O lado esquerdo foi acometido em oito casos, enquanto o direito apenas em três.

A transferência do músculo tríceps para bíceps proporcionou bons resultados em 10 pacientes, dos quais oito adquiriram força de flexão do cotovelo grau 4 (Figura 4), e dois força grau 3. Em um caso o resultado foi insatisfatório, cujo paciente adquiriu força de flexão grau 2. Dois pacientes evoluíram com complicações. Em um caso foi indicado fasciotomia, devido à suspeita de quadro inicial de síndrome compartimental, evoluindo satisfatoriamente com resultado final de força grau 3 de flexão do cotovelo. Houve necessidade de reoperação em outro caso, devido ao insuficiente tensionamento durante a sutura no bíceps. Este paciente também evoluiu com força grau 3 (Tabela 2).

\section{DISCUSSÃO}

Os pacientes portadores de lesão do plexo braquial apresentam quadro clínico extremamente variável, pois estas lesões podem determinar desde a perda parcial e transitória da sensibilidade no membro superior afetado, até a ausência total e permanente da sensibilidade e da motricidade, nestes casos geralmente devido à avulsão completa das raízes.

Tendo em vista a diversidade de possibilidades de le-

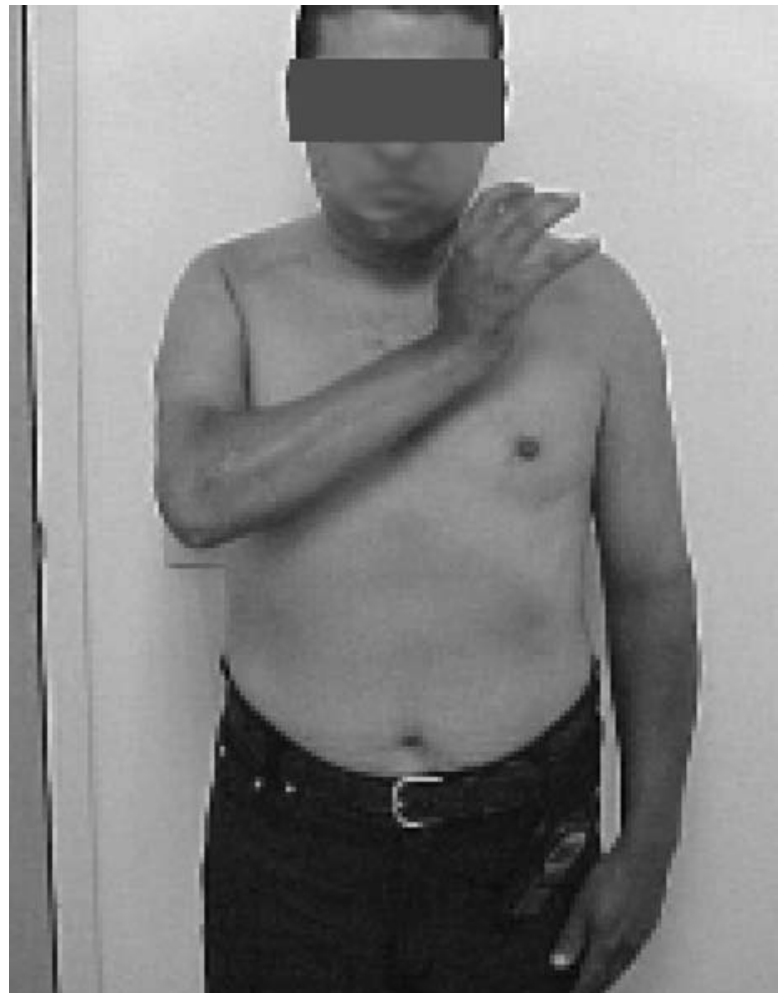

Figura 4 - Paciente apresentando força de flexão do cotovelo grau 4

Tabela 2 - Relação dos pacientes, idade, sexo, lado acometido, tipo de acidente, tempo de lesão até o procedimento e força muscular final

\begin{tabular}{c|c|c|c|c|c|c|c}
\hline Caso & Iniciais & Idade & Sexo & Lado & $\begin{array}{c}\text { Tipo de } \\
\text { acidente }\end{array}$ & $\begin{array}{c}\text { Tempo de } \\
\text { lesão até } \\
\text { cirurgia }\end{array}$ & $\begin{array}{c}\text { Força } \\
\text { muscular }\end{array}$ \\
\hline 1 & A.R.S. & $41 \mathrm{a}$ & $\mathrm{M}$ & $\mathrm{E}$ & Moto & $4 \mathrm{a} 3 \mathrm{~m}$ & $\mathrm{G} 4$ \\
\hline 2 & J.C.S.T. & $38 \mathrm{a}$ & $\mathrm{M}$ & $\mathrm{E}$ & Moto & $6 \mathrm{a} 2 \mathrm{~m}$ & $\mathrm{G} 4$ \\
\hline 3 & E.F.S. & $25 \mathrm{a}$ & $\mathrm{M}$ & $\mathrm{E}$ & Moto & $2 \mathrm{a} 11 \mathrm{~m}$ & $\mathrm{G} 4$ \\
\hline 4 & E.S.O. & $31 \mathrm{a}$ & $\mathrm{M}$ & $\mathrm{E}$ & Moto & $2 \mathrm{a} 8 \mathrm{~m}$ & $\mathrm{G} 4$ \\
\hline 5 & A.L.A. & $33 \mathrm{a}$ & $\mathrm{M}$ & $\mathrm{E}$ & Moto & $3 \mathrm{a} 4 \mathrm{~m}$ & $\mathrm{G} 4$ \\
\hline 6 & F.A.C. & $28 \mathrm{a}$ & $\mathrm{M}$ & $\mathrm{D}$ & Moto & $2 \mathrm{a}$ & $\mathrm{G} 4$ \\
\hline 7 & B.D.R.A. & $28 \mathrm{a}$ & $\mathrm{M}$ & $\mathrm{E}$ & Moto & $1 \mathrm{a} 9 \mathrm{~m}$ & $\mathrm{G} 4$ \\
\hline 8 & F.B.S. & $49 \mathrm{a}$ & $\mathrm{M}$ & $\mathrm{E}$ & $\begin{array}{c}\text { Queda } \\
\text { de altura }\end{array}$ & $6 \mathrm{a}$ & $\mathrm{G} 4$ \\
\hline 9 & J.C.M. & $47 \mathrm{a}$ & $\mathrm{M}$ & $\mathrm{E}$ & Moto & $3 \mathrm{a} 11 \mathrm{~m}$ & $\mathrm{G} 3$ \\
\hline 10 & F.R.S. & $24 \mathrm{a}$ & $\mathrm{M}$ & $\mathrm{D}$ & Moto & $3 \mathrm{a} 8 \mathrm{~m}$ & $\mathrm{G} 3$ \\
\hline 11 & S.A.S. & $27 \mathrm{a}$ & $\mathrm{F}$ & $\mathrm{D}$ & Moto & $2 \mathrm{a} 1 \mathrm{~m}$ & $\mathrm{G} 4$ \\
\hline
\end{tabular}

Fonte: Grupo de Mão e Microcirurgia - IOT-FMUSP

são e de apresentação clínica, os cirurgiões devem realizar avaliações minuciosas e registrar os territórios somáticos e a força muscular de todos os grupos musculares ${ }^{(9)}$.

A incapacidade de flexão do cotovelo ocasiona importante limitação funcional do membro, especialmente nas lesões isoladas do tronco superior, nas quais geralmente os movimentos da mão estão preservados. 
Concordamos com a maioria dos cirurgiões em eleger a flexão do cotovelo a prioridade número um no plano de tratamento.

A intervenção cirúrgica na fase aguda é a ideal, pois pode-se realizar neurólises, suturas término-terminais, interposições de enxertos e neurotizações ${ }^{(10)}$, buscandose a reinervação dos músculos-alvo.

Nos casos crônicos, a flexão do cotovelo pode ser restaurada pelas transferências musculotendíneas. Entre estas possibilidades, pode-se relacionar as transposições dos músculos grande dorsal ${ }^{(5)}$, peitoral maior ${ }^{(11)}$, peitoral menor, coracobraquial ${ }^{(9,12)}$, tríceps ${ }^{(6,7,13)}$, e flexoplastia de Staindler ${ }^{(3,4,13)}$, além dos transplantes musculares, destacando-se o grácil $^{(9,14)}$.

Azze et $a^{(11)}$ descreveram bons resultados com as transposições do peitoral maior e do grande dorsal, ressaltando que o segundo apresenta melhor resultado cosmético, sendo a primeira opção indicada para pacientes do sexo feminino.

Pardini et al $^{(13)}$ compararam as transposições do tríceps e dos músculos flexopronadores. Ressaltam a importância da seleção dos pacientes para a indicação da tenoplastia do tríceps, pois nos casos de pacientes que utilizam muletas, os cadeirantes e os que necessitam da extensão do cotovelo nos hobbies, esta técnica é contraindicada, obtendo-se melhores resultados com a transposição do tríceps.

Rostoucher et $a l^{(2)}$, em sua série de três casos, relataram bons resultados com transposição do tríceps, obtendo forças musculares graus 4 e 5, enquanto Hoang et $a l^{(6)}$, obtiveram bons resultados nos sete pacientes operados.

Rühmann et al $^{(7)}$, demonstraram bons resultados em 10 casos, nos quais obtiveram forças de flexão do cotovelo entre os graus 3 e 5 .

Estes resultados são semelhantes aos obtidos neste estudo, pois, dos 11 pacientes operados, oito adquiriram força de flexão grau 4, dois grau 3 e um caso de força grau 2.

A transposição anterior do tríceps é uma importante alternativa na restauração da flexão do cotovelo, pois o procedimento é relativamente simples, seguro e proporciona bons resultados funcionais e de avaliação satisfatória por parte dos pacientes.

A principal desvantagem desta técnica é a ausência de extensão ativa do cotovelo, em que a mesma é realizada pela gravidade. Embora este desconforto seja considerável, não contraindica o procedimento, visto que todos os pacientes ficaram satisfeitos com o resultado.

\section{CONCLUSÃO}

A transposição anterior do músculo tríceps proporcionou satisfação dos pacientes em todos os casos, exceto um, obtendo-se forças grau 4 em oito casos, grau 3 em dois casos e grau 2 em um. Nos casos de lesão crônica do tronco superior do plexo braquial, há viabilidade da indicação cirúrgica desta técnica.

\section{REFERÊNCIAS}

1. Abraciclo. Associação Brasileira dos Fabricantes de Motocicletas, Ciclomotores, Motonetas, Bicicletas e Similares. Disponível em: www.abraciclo.com.br. Acessado em 06-12-2009.

2. Rostoucher P, Alnot JY, Touam C, Oberlin C. Tendon transfers to restore elbow flexion after traumatic paralysis of the brachial plexus in adults. Int Orthop. 1998;22(4):255-62.

3. Monreal R. Steindler flexorplasty to restore elbow flexion in C5-C6-C7 brachial plexus palsy type. J Brachial Plex Peripher Nerve Inj. 2007;2:15.

4. Liu TK, Yang RS, Sun JS. Long-term results of the Steindler flexorplasty. Clin Orthop Relat Res. 1993;(296):104-8.

5. Gousheh J, Arab H, Gilbert A. The extended latissimus dorsi muscle island flap for flexion or extension of the fingers. J Hand Surg Br. 2000;25(2):160-5.

6. Hoang PH, Mills C, Burke FD. Triceps to biceps transfer for established brachial plexus palsy. J Bone Joint Surg Br. 1989;71(2):268-71.

7. Rühmann $\mathrm{O}$, Wirth $\mathrm{CJ}$, Gossé F. Triceps to biceps transfer to restore elbow flexion in three patients with brachial plexus palsy. Scand J Plast Reconstr Surg Hand Surg. 2000;34(4):355-62.

8. Doi K, Sakai K, Kuwata N, Ihara K, Kawai S. Double free-muscle transfer to restore prehension following complete brachial plexus avulsion. J Hand Surg Am.1995;20(3):408-14.

9. Rühmann O, Schmolke S, Gossé F, Wirth CJ. Transposition of local muscles to restore elbow flexion in brachial plexus palsy. Injury. 2002;33(7):597-609.

10. Seddon $\mathrm{HJ}$. Peripheral nerve injuries. In: Seddon $\mathrm{HJ}$, editor. Medical research council special report series $n^{\circ}$ 282. Part 1 . Methods of investigating nerve injuries. Nerve injuries Committee of the Medical Research Council. London: Stationary Office;1954. p. 1-15.

11. Azze RJ, Ferreira MC, Zumiotti AV. Transferência de músculo para restauração da flexão do cotovelo. Rev Bras Ortop. 1985;20(6):261-6.

12. Comtet JJ, Herzberg G, Naasan IA. Biomechanical basis of transfers for shoulder paralysis. Hand Clin. 1989;5(1):1-14.

13. Pardini AG, Freitas AL, Freitas AD, Tavares KE. Transferências tendinosas para flexão do cotovelo. Rev Bras Ortop. 1996;31(3):211-6.

14. Doi K. Management of total paralysis of the brachial plexus by the doublé freemuscle transfer technique. J Hand Surg Eur. 2008;33(3):240-51. 